\title{
PRODUCTS OF COMMUTATIVE RINGS AND ZERO-DIMENSIONALITY
}

\author{
ROBERT GILMER AND WILLIAM HEINZER
}

\begin{abstract}
If $R$ is a Noetherian ring and $n$ is a positive integer, then there are only finitely many ideals $I$ of $R$ such that the residue class ring $R / I$ has cardinality $\leq n$. If $R$ has Noetherian spectrum, then the preceding statement holds for prime ideals of $R$. Motivated by this, we consider the dimension of an infinite product of zero-dimensional commutative rings. Such a product must be either zero-dimensional or infinite-dimensional. We consider the structure of rings for which each subring is zero-dimensional and properties of rings that are directed union of Artinian subrings. Necessary and sufficient conditions are given in order that an infinite product of zero-dimensional rings be a directed union of Artinian subrings.
\end{abstract}

\section{INTRODUCTION}

All rings we consider are commutative and have a unity element. If $R$ is a subring of $S$ we assume the unity of $S$ is contained in $R$, and hence is the unity of $R$. We use $\operatorname{dim} R$ to denote the (Krull) dimension of a ring $R$, and $|S|$ to denote the cardinality of a set $S$.

If $n$ is a positive integer, if $\left\{I_{\alpha}\right\}$ is the set of ideals $I_{\alpha}$ of a ring $R$ such that $\left|R / I_{\alpha}\right| \leq n$, and if $I=\bigcap_{\alpha} I_{\alpha}$, then we prove (in Theorem 2.2) that: (a) either $R / I=(0)$ or $\operatorname{dim} R / I=0$, (b) if $R$ is Noetherian, then the set $\left\{I_{\alpha}\right\}$ is finite, (c) if $R$ has Noetherian spectrum, then the set $\left\{I_{\alpha}\right\}$ contains only finitely many prime ideals. The proof of Theorem 2.2 led us into consideration of the main themes of this paper: the dimension of a direct product $\prod_{\alpha} R_{\alpha}$ of commutative rings and of its subrings, particularly in the case where each $R_{\alpha}$ is zero-dimensional.

We use $N(R)$ and $J(R)$, respectively, to denote the nilradical and Jacobson radical of the ring $R$. If $x \in N(R)$ we define $\eta(x)=k$ if $x^{k}=0$ but $x^{k-1} \neq 0$, and we define the index of nilpotency of the ring $R$, denoted $\eta(R)$, to be $\sup \{\eta(x): x \in N(R)\}$. In Theorem 3.4 we show that if $\left\{R_{\alpha}\right\}_{\alpha \in A}$ is a family of zero-dimensional rings and $R=\prod_{\alpha} R_{\alpha}$, then the following conditions

Received by the editors June 26, 1989 and, in revised form, March 8, 1990.

1980 Mathematics Subject Classification (1985 Revision). Primary 13A99; Secondary 13A15, 13B02, $13 \mathrm{E} 05$.

Key words and phrases. Products of commutative rings, zero-dimensionality, ideals of finite norm, directed union of Artinian subrings, Noetherian ring, Noetherian spectrum, finite spectrum, hereditarily Noetherian ring, Krull dimension.

The first author's research was supported by NSF Grant DMS-8501003.

The second author's research was supported by NSF Grant DMS-8521767. 
are equivalent: (1) $\operatorname{dim} R=0$, (2) $J(R)=N(R)$, (3) $N(R)=\prod_{\alpha} N\left(R_{\alpha}\right)$, (4) there exists a positive integer $k$ such that $\left\{\alpha \in A: \eta\left(R_{\alpha}\right)>k\right\}$ is finite, (5) $\operatorname{dim} R \neq \infty$.

In $\S 4$ we consider conditions under which each subring of a ring $S$ is zero-dimensional. A ring $S$ with this property is said to be hereditarily zerodimensional. We also consider this zero-dimensionality condition in a relative context: if $R$ is a subring of $S$, we say that $(R, S)$, is a zero-dimensional pair if each subring of $S$ containing $R$ is zero-dimensional. Corollary 4.3 states that a ring $R$ is hereditarily zero-dimensional if and only if for each prime ideal $P$ of $R$, the ring $R / P$ is an absolutely algebraic field of nonzero characteristic. In Theorem 4.9 it is shown that if $\left\{R_{\alpha}\right\}_{\alpha \in A}$ is a family of rings, then $R=\prod_{\alpha} R_{\alpha}$ is hereditarily zero-dimensional if and only if each $R_{\alpha}$ is hereditarily zero-dimensional and there exists a positive integer $n$ such that the set $B=\left\{\alpha \in A: \eta\left(R_{\alpha}\right)>n\right.$ or there exists $P \in \operatorname{Spec}\left(R_{\alpha}\right)$ with $\left.\left|R_{\alpha} / P\right|>n\right\}$ is a finite set. In Theorem 4.10 we classify the hereditarily zero-dimensional rings that are hereditarily Noetherian as rings $R$ of the form $R=R_{1} \oplus \cdots \oplus R_{n} \oplus S$, where each $R_{i}$ is an absolutely algebraic field of nonzero characteristic and $S$ is a finite ring, or alternatively as rings $R$ for which $N(R)$ is finite and $R / N(R)$ is a finite direct sum of absolutely algebraic fields of nonzero characteristic.

In $\S 5$ we consider the structure of rings that are the directed union of Artinian subrings. Corollary 5.5 implies that if $(S, M)$ is a zero-dimensional quasilocal ring, then there exists an Artinian subring $R$ of $S$ such that $R \subseteq S$ is a zerodimensional pair. In particular, $S$ is a directed union of Artinian subrings. We present, in Example 5.6, an example of a ring $R$ that is the directed union of Artinian subrings but is such that $R$ is not integral over any subring with Noetherian spectrum.

$\S 6$ deals with the question of conditions under which a direct product of rings is expressible as a directed union of Artinian subrings, or more generally, of subrings with finite spectra. Theorem 6.7 states that if $\left\{R_{\alpha}\right\}_{\alpha \in A}$ is a family of rings and $T=\prod_{\alpha} R_{\alpha}$, then $T$ is a directed union of Artinian subrings if and only if each $R_{\alpha}$ is so expressible and there exists a positive integer $n$ such that the set $\left\{\alpha \in A: \eta\left(R_{\alpha}\right)>n\right.$ or $R_{\alpha}$ has a residue field $K_{\alpha}$ with $\left.\left|K_{\alpha}\right|>n\right\}$ is a finite set. There is an analogous result concerning rings that are a directed union of subrings with finite spectra. We conclude the paper with several questions concerning directed unions of subrings.

We use $\mathbb{Z}$ to denote the ring of integers, $\mathbb{Z}^{+}$to denote the positive integers, and $\mathbb{Q}$ to denote the field of rational numbers.

\section{IDEALS OF FINITE NORM IN A NOETHERIAN RING}

If $I$ is an ideal of a ring $R$, we denote by $n(I)$ the cardinality of the residue class ring $R / I \quad(n(I)=\infty$ if $R / I$ is infinite); $n(I)$ is called the norm of $I$ [BW, $\mathrm{G}_{1}, \mathrm{G}_{4}, \mathrm{p}$. 466]. Ideals of finite norm and rings $R$ such that $n(I)$ is finite for each nonzero ideal $I$ of $R$ have historically commanded strong interest. (Chew and Lawn [CL] and Levitz and Mott [LM] have considered the class of such rings. We follow the terminology of [CL] and call such rings residually finite.) The reason for this historical interest stems from algebraic number theory. For example, any subring of a finite algebraic number field (or, more generally, of a global field) is residually finite, and in fact, since residually 
finite rings are Noetherian, it follows from results of $\left[G_{2}\right]$ that global fields are characterized by the property that each of their subrings is residually finite. More recently, ideals of finite norm have been shown to play an important role in the study of rings of integer-valued polynomials of an integral domain $D$, particularly in the case where $D$ is Noetherian. (See, for example, [CC, C, Mc, and SSY].) In this section we consider the following question: If $R$ is a Noetherian ring and $k$ is a fixed positive integer, is the set of ideals $I$ of $R$ such that $n(R)=k$ finite? We provide an affirmative answer to this question in Theorem 2.2.

Proposition 2.1. Let $\mathscr{R}=\left\{R_{\alpha}\right\}$ be a family of finite rings such that the elements of $\mathscr{R}$ lie in a finite number of isomorphism classes. Let $R=\prod R_{\alpha}$ and denote by $\pi$ the prime subring of $R$. There exists a monic polynomial $h(X) \in \mathbb{Z}[X]$ that vanishes identically on $R$. Hence $R$ is integral over $\pi$, and each subring of $R$ containing $\pi$ is zero-dimensional.

Proof. We prove the assertion concerning $h(X)$ first for a finite ring $S=$ $\left\{s_{i}\right\}_{i=1}^{t}$. Thus, if $s \in S$, the powers $s, s^{2}, \ldots$ are not all distinct, so there exists a monic polynomial $h_{s}(X)=X^{m_{s}}-X^{n_{s}} \in \mathbb{Z}[X]$ with $s$ as a root. Hence $h(X)=\prod_{i=1}^{t} h_{s^{i}}(X) \in \mathbb{Z}[X]$ is monic and vanishes on $S$.

In the problem at hand, partition $\mathscr{R}$ according to its isomorphism classes$\mathscr{R}=\bigcup_{i=1}^{t} \mathscr{R}^{(i)}$-and choose $R_{\alpha_{i}} \in \mathscr{R}^{(i)}$ for each $i$. By the preceding paragraph, there exists a monic polynomial $h_{i}(x) \in \mathbb{Z}[X]$ that vanishes identically on $R_{\alpha_{i}}$, and hence on each element of $\mathscr{R}^{(i)}$. Consequently, the monic polynomial $h(X)=\prod_{i=1}^{t} h_{i}(X) \in \mathbb{Z}[X]$ vanishes identically on $R$. It follows that $R$ is integral over $\pi$. Because the set $\left\{\left|R_{\alpha}\right|\right\}$ is bounded, $R$ has nonzero characteristic. Hence $\pi$ is finite and $\operatorname{dim} S=0$ for each subring $S$ of $R$ containing $\pi$.

We remark that if $\mathscr{R}=\left\{R_{\alpha}\right\}$ is a family of finite rings, then there are only finitely many isomorphism classes of elements of $\mathscr{R}$ if and only if $\left\{\left|R_{\alpha}\right|\right\}$ is bounded; this is true since rings of different cardinalities are not isomorphic and since, for a fixed positive integer $n$, there are only finitely many isomorphism classes of rings of cardinality $n$.

We observe that under the hypothesis of Proposition 2.1, each subring of $R$, even one without an identity element, has dimension $\leq 0$. To see this, we note that if $T$ is a ring with identity element $e$ and if (1) each subring of $T$ containing $e$ has dimension $\leq m$, then (2) each subring of $T$ has dimension $\leq m$, by the following argument: If $S$ is an arbitrary subring of $T$ and if $S^{*}=S+\mathbb{Z} e$, then $\operatorname{dim} S \leq \operatorname{dim} S^{*}$ [AG, Corollary 3.4], and hence $\operatorname{dim} S \leq m$.

Theorem 2.2. Let $R$ be a ring, let $m \in \mathbb{Z}^{+}$, let $\mathscr{I}_{m}=\left\{I_{\alpha}\right\}$ be the set of ideals $I_{\alpha}$ of $R$ such that $\left|R / I_{\alpha}\right| \leq m$, and let $I=\bigcap_{\alpha} I_{\alpha}$. Then

(a) Either $R / I=(0)$, or else $R / I$ is zero-dimensional.

(b) If $R$ is Noetherian, $\mathscr{I}_{m}$ is finite.

(c) If $R$ has Noetherian spectrum, then $\mathscr{I}_{m}$ contains only finitely many prime ideals.

Proof. The proof of Theorem 2.2 is trivial if $\mathscr{I}_{m}=\{R\}$, so we assume throughout the proof that $\mathscr{I}_{m}$ contains a proper ideal.

(a) The diagonal imbedding $\varphi$ of $R$ into $\prod_{\alpha}\left(R / I_{\alpha}\right)$ has kernel $I$ and $\varphi(R)$ 
contains the prime subring of $\prod_{\alpha}\left(R / I_{\alpha}\right)$. Hence Proposition 2.1 implies that $\operatorname{dim}(R / I)=\operatorname{dim} \varphi(R)=0$.

(b) If $R$ is Noetherian, then $R / I$ is zero-dimensional Noetherian, and hence is the direct sum of a finite family $\left\{\left(R_{i}, M_{i}\right)\right\}_{i=1}^{n}$ of Noetherian primary rings [ZS, Chapter IV, §3]. We have $\bigcap_{\alpha}\left(I_{\alpha} / I\right)=(0)$, so given $j$, there exists $I_{\alpha_{j}} \in \mathscr{I}_{m}$ such that $R_{j} \nsubseteq I_{\alpha_{j}} / I$. We write $I_{\alpha_{j}} / I=B_{1} \oplus \cdots \oplus B_{n}$, where $B_{i}$ is an ideal of $R_{i}$ and $B_{j}<R_{j}$. Then $(R / I) /\left(I_{\alpha_{j}} / I\right) \simeq R / I_{\alpha_{j}} \simeq\left(R_{1} / B_{1}\right) \oplus \cdots \oplus\left(R_{n} / B_{n}\right)$ is a finite ring, so in particular, $R_{j} / B_{j}$ is finite. Because $B_{j}<R_{j}$, it follows that $R_{j} / M_{j}$ is finite, and since $R_{j}$ is Noetherian and primary, an inductive argument on the order of nilpotence of $M_{j}$ easily shows that $R_{j}$ is finite. Hence $R / I$ is finite and has only finitely many ideals. Because the map $I_{\alpha} \rightarrow I_{\alpha} / I$ is an injection of $\mathscr{I}_{m}$ into the family of ideals of $R / I$, we conclude that $\mathscr{I}_{m}$ is finite.

(c) Suppose $R$ has Noetherian spectrum. Then any prime ideal $P \in \mathscr{I}_{m}$ is a minimal prime of $I$ since $\operatorname{dim}(R / I)=0$. Because $I$ has only finitely many minimal primes, it follows that $\mathscr{I}_{m} \cap \operatorname{Spec}(R)$ is finite, as asserted. This completes the proof of Theorem 2.2.

We remark that the conclusion of (b) fails badly if $R$ is assumed merely to have Noetherian spectrum. For example, if $F$ is a finite field with $q$ elements and if $W$ is an infinite-dimensional vector space over $F$, then the idealization $R=F(+) W$ of $F$ and $W$ is a zero-dimensional quasilocal ring with infinitely many ideals of norm $q^{n}$ for each $n>1$. In this example, the intersection of the family of ideals of $R$ of norm $q^{n}$ is (0) for each $n>1$. We note that if $D$ is the polynomial ring $F\left[\left\{X_{\alpha}\right\}\right]$, where $\left|\left\{X_{\alpha}\right\}\right|=\operatorname{dim} W$, then $R \simeq D / M^{2}$, where $M=\left\{X_{\alpha}\right\} D$.

\section{The KRUll dimension of $\prod R_{\alpha}$}

Proposition 2.1 shows that if $\left\{R_{\alpha}\right\}$ is a family of finite rings and if the set $\left\{\left|R_{\alpha}\right|\right\}$ is bounded, then $\operatorname{dim} \prod R_{\alpha}=0$. In this section we consider the problem of determining $\operatorname{dim} S$, where $S=\prod S_{\alpha}$ is the product of an arbitrary family $\left\{S_{\alpha}\right\}_{\alpha \in A}$ of rings. Theorem 3.5 gives a complete answer to this problem. In connection with the problem, it is clear that $\operatorname{dim} S \geq \sup \left\{\operatorname{dim} S_{\alpha}\right\}$, so equality holds if the set $\left\{\operatorname{dim} S_{\alpha}\right\}$ is unbounded; of course, it is well known that $\operatorname{dim} S=$ $\sup \left\{\operatorname{dim} S_{\alpha}\right\}_{\alpha \in A}$ if the set $A$ is finite. An important case to consider in the determination of $\operatorname{dim} \prod S_{\alpha}$ is that in which each $S_{\alpha}$ is zero-dimensional, and there is a known result, due to Maroscia [M, Proposition 2.6], in this case. We record this result below as Proposition 3.1; recall that $N(\cdot)$ denotes the nilradical.

Proposition 3.1 (Maroscia [M]). If $S=\prod S_{\alpha}$ is a product of zero-dimensional rings $S_{\alpha}$, then $S$ is zero-dimensional if and only if $N(S)=\prod N\left(S_{\alpha}\right)$ or, equivalently, if and only if $N(S) \supseteq \prod N\left(S_{\alpha}\right)$.

A special case of Proposition 3.1 is the basic result that an arbitrary product of zero-dimensional reduced rings (that is, von Neumann regular rings) is von Neumann regular, and hence is zero-dimensional. In Theorem 3.4 we show that $\operatorname{dim} \prod S_{\alpha} \in\{0, \infty\}$ if each $S_{\alpha}$ is zero-dimensional.

Proposition 3.2. Suppose $\left\{R_{\alpha}\right\}_{\alpha \in A}$ is a family of rings, each of dimension $\geq k$, where $k \geq 1$ and $A$ is infinite. If $R=\prod_{\alpha} R_{\alpha}$, then $\operatorname{dim} R \geq k+1$. 
Proof. For $\alpha \in A$, let $D_{\alpha}$ be a domain of dimension $\geq k$ that is a homomorphic image of $R_{\alpha}$. Since $\prod_{\alpha} D_{\alpha}$ is a homomorphic image of $R$, we may assume that $R_{\alpha}=D_{\alpha}$ for each $\alpha$.

The conclusion holds if $\operatorname{dim} D_{\alpha}>k$ for some $\alpha$, so we assume without loss of generality that $\operatorname{dim} D_{\alpha}=k$ for each $\alpha$. Also, we may assume that $A=$ $\mathbb{Z}^{+}$, for if $B$ is a countably infinite subset of $A$, then $\operatorname{dim} R \geq \operatorname{dim} \prod_{\alpha \in B} D_{\alpha}$ since $\prod_{\alpha \in B} D_{\alpha}$ is a homomorphic image of $R$. Hence, for $R=\prod_{i=1}^{\infty} D_{i}$ and $\operatorname{dim} D_{i}=k$ for each $i$, we prove the result by induction on $k$.

If $k=1$, choose for each $i$ a nonzero nonunit, $d_{i} \in D_{i}$ and let $r=\left\{d_{i}^{i}\right\}_{1}^{\infty} \in$ $R$. Then $r$ is a regular element of $R$, and hence $\operatorname{dim} R \geq \operatorname{dim}(R /(r))+1$. Now $R /(r) \simeq \prod_{1}^{\infty}\left(D_{i} /\left(d_{i}^{i}\right)\right)$, where $D_{i} /\left(d_{i}^{i}\right)$ is zero-dimensional. The element $\left\{d_{i}+\left(d_{i}^{i}\right)\right\}_{i=1}^{\infty}$ belongs, however, to $\prod_{1}^{\infty} N\left(D_{i} /\left(d_{i}^{i}\right)\right)$ but not to $N\left(\prod_{1}^{\infty}\left(D_{i} /\left(d_{i}^{i}\right)\right)\right)$, and hence $\operatorname{dim}(R /(r))>0$ by Proposition 3.1. Consequently, $\operatorname{dim} R>1$ and the desired conclusion holds for $k=1$. At the inductive step, we assume that $\operatorname{dim} D_{i}=k$ for each $i$, where $k>1$, and we assume that the result is true for domains of dimension $k-1$. For each $i$, choose a prime ideal $P_{i}$ of $D_{i}$ such that $\operatorname{dim}\left(D_{i} / P_{i}\right)=k-1$ and choose a nonzero element $x_{i} \in P_{i}$. Again $x=\left\{x_{i}\right\}_{1}^{\infty}$ is a regular element of $R$, and hence $\operatorname{dim} R \geq \operatorname{dim}(R /(x))+1$. But $R /(x) \simeq \prod_{1}^{\infty}\left(D_{i} /\left(x_{i}\right)\right)$ has as a homomorphic image the ring $\prod_{1}^{\infty}\left(D_{i} / P_{i}\right)$, which has dimension $\geq k$ by the induction hypothesis. Therefore $\operatorname{dim} R \geq k+1$, and Proposition 3.2 follows.

Theorem 3.3. Suppose $\left\{R_{\alpha}\right\}_{\alpha \in A}$ is a family of rings and $R=\prod_{\alpha} R_{\alpha}$. If infinitely many of the rings $R_{\alpha}$ have positive dimension, then $R$ is infinite-dimensional. ${ }^{1}$

Proof. As in the proof of Proposition 3.2, there is no loss of generality in assuming that $A=\mathbb{Z}^{+}$and that each $R_{i}=D_{i}$ is an integral domain of positive dimension. To prove Theorem 3.3, we first establish by induction the following assertion: For each positive integer $k, R$ has a homomorphic image $E^{(k)}=\prod_{i=1}^{\infty} E_{i}^{(k)}$, where each $E_{i}^{(k)}$ is a domain of dimension $\geq k$. For $k=1$, we can take $E^{(1)}=R$. We assume that the assertion is valid for a fixed integer $t \geq 1$. Partition $\mathbb{Z}^{+}$into an infinite number of infinite subsets: $\mathbb{Z}^{+}=\bigcup_{i=1}^{\infty} W_{i}$. Then $R=\prod_{i=1}^{\infty} R_{i}$, where $R_{i}=\prod_{j \in W_{i}} D_{j}$. Applying the induction hypothesis to $R_{i}$, we conclude that $R_{i}$ admits a homomorphic image that is the direct product of a countably infinite family of domains of dimension $\geq t$, and hence, by Proposition 3.2, $\operatorname{dim} R_{i} \geq t+1$. Let $E_{i}^{(t+1)}$ be a domain of dimension $\geq t+1$ that is a homomorphic image of $R_{i}$. Then $E^{(t+1)}=\prod_{1}^{\infty} E_{i}^{(t+1)}$ is a homomorphic image of $R=\prod_{1}^{\infty}, R_{i}$, and hence the assertion follows by induction. It is clear that the assertion implies that $\operatorname{dim} R$ is infinite.

For the statement of Theorem 3.4, we use the following notation. If $S$ is a ring and $x \in N(S)$, we denote by $\eta(x)$ the index of nilpotency of $x$-that is, $\eta(x)=k$ if $x^{k}=0$ but $x^{k-1} \neq 0$. We define $\eta(S)$ to be $\sup \{\eta(x): x \in N(S)\}$; if the set $\{\eta(x): x \in N(S)\}$ is unbounded, then we write $\eta(S)=\infty$. Theorem 3.4 is an extension of Proposition 3.1; recall that $J(\cdot)$ denotes the Jacobson radical.

\footnotetext{
${ }^{1}$ In 1963 , J. Keisler showed the first author a proof that $\mathbb{Z}^{\omega}$, the countably infinite product of $\mathbb{Z}$ with itself, is infinite-dimensional. To our knowledge, this proof has not appeared in the literature. Keisler's proof, which used ultrafilters, seems to have been disjoint from the proof given here.
} 
Theorem 3.4. Suppose $\left\{R_{\alpha}\right\}_{\alpha \in A}$ is a family of zero-dimensional rings and let $R=\prod R_{\alpha}$. The following conditions are equivalent. ${ }^{2}$

(1) $\operatorname{dim} R=0$.

(2) $J(R)=N(R)$.

(3) $N(R)=\prod N\left(R_{\alpha}\right)$.

(4) There exists $k \in \mathbb{Z}^{+}$such that $\left\{\alpha \in A: \eta\left(R_{\alpha}\right)>k\right\}$ is finite.

(5) $\operatorname{dim} R \neq \infty$.

Proof. The equivalence of (1) and (3) is the content of Proposition 3.1, and the implications $(1) \Rightarrow(2)$ and $(1) \Rightarrow(5)$ are patent. That (2) implies (3) follows from the fact that $J(R)=\prod J\left(R_{\alpha}\right) \supseteq \prod N\left(R_{\alpha}\right)$. To complete the proof, we show that (3) and (4) are equivalent and that (5) fails if (4) fails.

(3) $\Rightarrow(4)$. If (4) fails, then there exist an infinite subset $\left\{\alpha_{i}\right\}_{1}^{\infty}$ of $A$ and elements $x_{\alpha_{i}} \in N\left(R_{\alpha_{i}}\right)$ such that $x_{\alpha_{i}}^{i} \neq 0$. Let $x=\left\{x_{\alpha}\right\} \in \prod N\left(R_{\alpha}\right)$, where $x_{\alpha}=0$ if $\alpha \in A \backslash\left\{\alpha_{i}\right\}_{1}^{\infty}$. Clearly $x^{i}=\left\{x_{\alpha}^{i}\right\} \neq 0$ for each $i$, and hence $x \notin N(R)$ and (3) also fails.

(4) $\Rightarrow(3)$. We need to show that (4) implies that $\prod N\left(R_{\alpha}\right) \subseteq N(R)$. Thus, let $x=\left\{x_{\alpha}\right\} \in \prod N\left(R_{\alpha}\right)$ and let $\left\{\alpha_{i}\right\}_{i=1}^{m}$ be the finite subset of $A$ consisting of elements $\alpha$ such that $\eta\left(R_{\alpha}\right)>k$. If $t=\sup \left\{k, \eta\left(x_{\alpha_{1}}, \ldots, \eta\left(x_{\alpha_{m}}\right)\right\}\right.$, it is clear that $x^{t}=\left\{x_{\alpha}^{t}\right\}=0$, that $x \in N(R)$, and hence that $\prod_{\alpha} N\left(R_{\alpha}\right) \subseteq N(R)$.

$(5) \Rightarrow(4)$. If (4) fails, we partition the set $A$ into a countably infinite family of subsets $A_{1}, A_{2}, \ldots$ such that, for all $i, k \in \mathbb{Z}^{+}$, the set $\left\{\alpha \in A_{i}\right.$ : $\left.\eta\left(R_{\alpha}\right)>k\right\}$ is infinite; this is easy to do via a traditional diagonal process. If $S_{i}=\prod_{\alpha \in A_{i}} R_{\alpha}$, then since (1) and (4) are equivalent, it follows that $\operatorname{dim} S_{i}>0$. Hence a homomorphic image $D_{i}$ of $S_{i}$ is an integral domain of dimension $\geq 1$, and $\prod_{1}^{\infty} D_{i}$ is a homomorphic image of $\prod_{1}^{\infty} S_{i}=R$. Theorem 3.3 shows that $\operatorname{dim} \prod D_{i}=\infty$, and hence $\operatorname{dim} R=\infty$ as well. This completes the proof of Theorem 3.4.

It follows from Corollary 8 of $\left[\mathrm{GH}_{4}\right]$ that the conditions of Theorem 3.4 are also equivalent to the condition that $R$ is imbeddable in a zero-dimensional ring. We record in Theorem 3.5 the complete answer to the problem of determining $\operatorname{dim}\left(\Pi R_{\alpha}\right)$ in the general case; Theorem 3.5 follows immediately from (3.3) and (3.4).

Theorem 3.5. Let $\left\{R_{\alpha}\right\}_{\alpha \in A}$ be a family of finite-dimensional rings, let $B=\{\alpha \in$ $\left.A: \operatorname{dim} R_{\alpha}>0\right\}$, let $C=A \backslash B$, and let $R=\prod R_{\alpha}$.

(1) $R$ is finite-dimensional if and only if $B$ is finite and there exists $k \in \mathbb{Z}^{+}$ such that $\left\{\gamma \in C: \eta\left(R_{\gamma}\right)>k\right\}$ is finite.

(2) If $\operatorname{dim} R$ is finite, then $\operatorname{dim} R=\sup \left\{\operatorname{dim} R_{\alpha}: \alpha \in A\right\}$.

We remark that an alternate way of expressing the equivalent conditions in (1) of Theorem 3.5 is to state that there exists $k \in \mathbb{Z}^{+}$such that the set $\left\{\alpha \in A: \operatorname{dim} R_{\alpha}>0\right\} \cup\left\{\alpha \in A: \eta\left(R_{\alpha}\right)>k\right\}$ is finite.

To conclude this section we record a corollary that follows from Theorem 3.5 and the proofs of Theorems 3.3 and 3.4.

\footnotetext{
${ }^{2}$ In a conversation with the second author in November 1983, M. Hochster and R. Wiegand outlined a proof that $\prod_{n=1}^{\infty}\left(\mathbb{Z} / p^{n} \mathbb{Z}\right), p$ a prime, is infinite-dimensional. The results presented here as Proposition 3.2, Theorem 3.3, and Theorem 3.5 were known to Hochster and Wiegand, but to our knowledge they have not appeared in print.
} 
Corollary 3.6. If $\left\{R_{\alpha}\right\}_{\alpha \in A}$ is a family of finite-dimensional rings, if $k \in \mathbb{Z}^{+}$, and if $R=\prod_{\alpha \in A} R_{\alpha}$ is infinite-dimensional, then $R$ admits a homomorphic image $\prod_{i=1}^{\infty} D_{i}$, where each $D_{i}$ is an integral domain of dimension $\geq k$.

\section{HEREDITARILY ZERO-DIMENSIONAL RINGS}

Motivated by its usefulness in the proof of Theorem 2.2, we consider in this section conditions under which each subring of a ring is zero-dimensional. We refer to a ring with this property as being hereditarily zero-dimensional. We also consider this zero-dimensionality condition in a relative context: if $R$ is a subring of $S$, we say that $(R, S)$ is a zero-dimensional pair if each subring of $S$ containing $R$ is zero-dimensional. Thus a ring $R$ is hereditarily zerodimensional if and only if $(\pi, R)$ is a zero-dimensional pair, where $\pi$ is the prime subring of $R$.

If $\operatorname{dim} R=0$ and if $S$ is an integral extension of $R$, then it is clear that $(R, S)$ is a zero-dimensional pair. To establish the converse, we use the following result.

Proposition 4.1. If $R$ is a subring of $S$, then $S$ is integral over $R$ if and only if for each prime ideal $Q$ of $S, S / Q$ is integral over $R /(Q \cap R)$.

Proof (cf. [ $\left[\mathrm{GH}_{2}\right.$, p. 227]). If $S / R$ is integral, the given condition clearly holds. Thus, we establish the converse.

For a fixed $s \in S$, let $G=\{f(s): f(X) \in R[X]$ is a monic polynomial $\}$. Then $G$ is a multiplicative system in $S$ and $s$ is integral over $R$ if and only if $0 \in G$. If $0 \notin G$, then there exists a prime ideal $Q$ of $S$ such that $Q \cap G=\varnothing$. It follows that the image of $s$ in $S / Q$ is not integral over $R /(Q \cap R)$.

Corollary 4.2. If $R$ is a zero-dimensional subring of the ring $S$, then $(R, S)$ is a zero-dimensional pair if and only if $S$ is integral over $R$. In particular, a ring $T$ is hereditarily zero-dimensional if and only if $\operatorname{char} T \neq 0$ and $T$ is integral over its prime subring.

Proof. Clearly $(R, S)$ is a zero-dimensional pair if $S / R$ is integral. Conversely, if $(R, S)$ is a zero-dimensional pair, then for each prime ideal $Q$ of $S,(R /(Q \cap R), S / Q)$ is a zero-dimensional pair of integral domains. And for integral domains $D \subseteq E$, it is clear that $(D, E)$ is a zero-dimensional pair if and only if $D$ and $E$ are fields and $E / D$ is algebraic. Therefore $S / Q$ is integral over $R /(Q \cap R)$ for each prime ideal $Q$ of $S$, and by Proposition 4.1, $S$ is integral over $R$.

Corollary 4.3. A ring $R$ is hereditarily zero-dimensional $\Leftrightarrow$ for each prime ideal $P$ of $R$, the ring $R / P$ is an absolutely algebraic field of nonzero characteristic.

Proof. $(\Rightarrow)$. This implication follows from Corollary 4.2 and Proposition 4.1.

$(\Leftarrow)$. The hypothesis implies that $R$ is zero-dimensional. Moreover, (4.1) shows that $R$ is integral over its prime subring $\pi$. Therefore $\pi$ is zerodimensional and char $R \neq 0$. Thus, by (4.2), $R$ is hereditarily zero-dimensional.

Remark 4.4. (i) If $R$ is a ring and $\operatorname{Spec}(R)=\left\{P_{\alpha}\right\}_{\alpha \in A}$, then $\operatorname{char} R \neq 0 \Leftrightarrow$ $\operatorname{char}\left(R / P_{\alpha}\right) \neq 0$ for each $\alpha$ and $\left\{\operatorname{char}\left(R / P_{\alpha}\right)\right\}_{\alpha \in A}$ is a finite set.

(ii) Suppose $R$ is a zero-dimensional ring with family $\left\{K_{\alpha}\right\}_{\alpha \in A}$ of residue fields. If the set 
is infinite, then $0 \in \mathscr{C}(R)$.

$$
\mathscr{C}(R)=\left\{\operatorname{char} K_{\alpha}: \alpha \in A\right\}
$$

If $\left\{K_{\alpha}\right\}_{\alpha \in A}$ is a family of fields, it would be interesting to have necessary and sufficient conditions in order that, up to isomorphism, $\left\{K_{\alpha}\right\}_{\alpha \in A}$ is the family of residue fields of a zero-dimensional ring $R$ (i.e., such that $\operatorname{Spec}(R)=\left\{P_{\alpha}\right\}_{\alpha \in A}$, where $R / P_{\alpha} \cong K_{\alpha}$ for each $\alpha$ ); part (ii) of (4.4) indicates that some restrictions on the family $\left\{K_{\alpha}\right\}$ are required. We have been able to show that if $\left\{K_{\alpha}\right\}_{\alpha \in A}$ is a family of subfields of a field and if the set $\left\{K_{\alpha}\right\}$ contains a finite subset $\left\{K_{i}\right\}_{i=1}^{n}$ such that each $K_{\alpha}$ contains some $K_{i}$, then there exists such a zerodimensional ring $R$, but the full story here remains to be explored.

Concerning the structure of zero-dimensional pairs, we observe the following.

Proposition 4.5. Assume that $R$ and $S$ are zero-dimensional rings, $R$ is a subring of $S$, and that $S$ is a finitely generated $R$-algebra. Then $(R, S)$ is a zero-dimensional pair.

Proof. We need to show that $S$ is integral over $R$, and to do so, it suffices, by (4.1), to show that $S / Q$ is integral over $R /(Q \cap R)$ for each $Q \in \operatorname{Spec}(S)$. Since $R$ and $S$ are zero-dimensional, $S / Q$ is a field that is a finitely generated algebra over the field $R / Q \cap R)$. By the field-theoretic form of Hilbert's Nullstellensatz [K, p. 16], $S / Q$ is algebraic, and hence integral, over $R /(Q \cap R)$.

If $K$ is a transcendental field extension of a field $F$ and if $A$ is a subset of $K$ such that $K=F[A]$, then it is known (see, for example, $\left[\mathrm{G}_{3}\right.$, Theorem 2.5]) that $|A|=|K|$. Replacing the reference to Hilbert's Nullstellensatz in the proof of Proposition 4.5 by this result, we easily obtain the following related result.

Proposition 4.6. Assume that $R$ and $S$ are zero-dimensional rings, that $R$ is a subring of $S$, and that $S$ is generated as an $R$-algebra by a set of cardinality $\beta$, where $\beta<|R / M|$ for each maximal ideal $M$ of $R$. Then $(R, S)$ is a zero-dimensional pair.

Remark 4.7. If $R$ is a zero-dimensional ring and $S$ is an extension ring of $R$, then we have seen that the following conditions (i)-(iv) are equivalent: (i) $(R, S)$ is a zero-dimensional pair; (ii) $S$ is integral over $R$; (iii) for each $Q \in \operatorname{Spec}(S), S / Q$ is integral over $R /(Q \cap R)$; (iv) $(R /(Q \cap R), S / Q)$ is a zero-dimensional pair for each $Q \in \operatorname{Spec}(S)$. Therefore the problem of determining whether $(R, S)$ is a zero-dimensional pair reduces in some sense to consideration of the case where $R=K$ is a field. Up to isomorphism we have, of course, that

$$
S=K\left[\left\{X_{\alpha}\right\}_{\alpha \in A}\right] / I,
$$

where $\left\{X_{\alpha}\right\}_{\alpha \in A}$ is a set of indeterminates over $K$ and $I$ is an ideal in the polynomial ring $K\left[\left\{X_{\alpha}\right\}_{\alpha \in A}\right]$. In this situation we have that $(K, S)$ is a zerodimensional pair if and only if $I \cap K[X] \neq(0)$ for each $X \in\left\{X_{\alpha}\right\}$.

We next turn to the problem of determining conditions under which the product of a family $\left\{R_{\alpha}\right\}_{\alpha \in A}$ of hereditarily zero-dimensional rings is again hereditarily zero-dimensional. Such need not be the case; for example, the ring $R=\prod_{i=1}^{\infty} G F\left(p_{i}\right)$, where $\left\{p_{i}\right\}_{i=1}^{\infty}$ is an infinite set of primes, has characteristic 0 , and therefore admits a one-dimensional subring. The next result records two basic properties that are related to the problem just mentioned. 
Lemma 4.8. Let $\{R\} \cup\left\{R_{i}\right\}_{i=1}^{n}$ be a finite collection of rings.

(i) If $R$ is hereditarily zero-dimensional, then so is each homomorphic image of $R$.

(ii) $\bigoplus_{i=1}^{n} R_{i}$ is hereditarily zero-dimensional if and only if each $R_{i}$ has this property.

Proof. Apply Corollary 4.3.

Theorem 4.9. Let $\left\{R_{\alpha}\right\}_{\alpha \in A}$ be a family of rings. The ring $R=\prod_{\alpha \in A} R_{\alpha}$ is hereditarily zero-dimensional if and only if each $R_{\alpha}$ is hereditarily zero-dimensional and there exists a positive integer $n$ such that the set $B=\left\{\alpha \in A: \eta\left(R_{\alpha}\right)>n\right.$ or there exists $P \in \operatorname{Spec} R_{\alpha}$ such that $\left.\left|R_{\alpha} / P\right|>n\right\}$ is a finite set.

Proof. $(\Rightarrow)$. Clearly each $R_{\alpha}$ is hereditarily zero-dimensional. Suppose there does not exist a positive integer $n$ such that the corresponding set $B$ is finite. Since $R$ is zero-dimensional, Theorem 3.4 then implies that there exists a countably infinite subset $\left\{\alpha_{i}\right\}_{i=1}^{\infty}$ of $A$ such that, for each $i$, there exists $P_{\alpha_{i}} \in \operatorname{Spec}\left(R_{\alpha_{i}}\right)$ such that $\left|R_{\alpha_{i}} / P_{\alpha_{i}}\right|>i$. Set $K_{i}=R_{\alpha_{i}} / P_{\alpha_{i}}$. Then $K_{i}$ is an absolutely algebraic field of nonzero characteristic, and since $S=\prod_{i=1}^{\infty} R_{\alpha_{i}}$ is a homomorphic image of $R$, the ring $S$ is hereditarily zero-dimensional. Part (ii) of Remark 4.4 shows that the set $\left\{\text { char } K_{i}\right\}_{i=1}^{\infty}$ is finite; hence by passage to an appropriate homomorphic image of $S$, we may assume without loss of generality that each $K_{i}$ has characteristic $p$ and that $\left|K_{i}\right|>p^{i}$ for each $i$. Choose, for each $i, a_{i} \in K_{i}$, such that the degree of $a_{i}$ over $G F(p)$ is greater than $i$. Then $a=\left(a_{1}, a_{2}, \ldots\right) \in S$ is transcendental over the prime subring $\pi \simeq G F(p)$ of $S$, contrary to the assumption that $S$ is hereditarily zero-dimensional. Thus, the set $B$ is finite.

$(\Leftarrow)$. Corollary 4.3 implies that $R$ is hereditarily zero-dimensional if and only if $R / N(R)$ is hereditarily zero-dimensional. By Theorem 3.4, $N(R)=$ $\prod_{\alpha \in A} N\left(R_{\alpha}\right)$, and hence $R / N(R) \simeq \prod_{\alpha \in A}\left(R_{\alpha} / N\left(R_{\alpha}\right)\right)$. Thus in proving that $R$ is hereditarily zero-dimensional, we may assume that each $R_{\alpha}$ is reduced. Let $C=\left\{\alpha_{1}, \ldots, \alpha_{m}\right\}$. Then $R=R_{\alpha_{1}} \oplus \cdots \oplus R_{\alpha_{m}} \oplus T$, where $T=\prod_{\alpha \in A \backslash C} R_{\alpha}$, and to show that $R$ is hereditarily zero-dimensional, it suffices, by Lemma 4.7, to show that $T$ is hereditarily zero-dimensional. Thus we assume without loss of generality that each $R_{\alpha}$ is reduced and that each of its residue fields has cardinality at most $n$. The reduced ring $R_{\alpha}$ is canonically imbedded in $\prod\left\{R_{\alpha} / P: P \in \operatorname{Spec} R_{\alpha}\right\}=S_{\alpha}$, and hence there exists a canonical injection of $R$ into the ring $\prod_{\alpha \in A} S_{\alpha}$. Proposition 2.1 shows that $\prod_{\alpha \in A} S_{\alpha}$ is hereditarily zero-dimensional, so $R$ has the same property, as we wished to show.

A ring $R$ is said to be hereditarily Noetherian if each subring of $R$ is Noetherian. In Theorem 4.10 we determine those hereditarily zero-dimensional rings that are hereditarily Noetherian.

Theorem 4.10. Let $R$ be a hereditarily zero-dimensional ring. The following conditions are equivalent.

(1) $R$ is hereditarily Noetherian.

(2) The nilradical $N(R)$ of $R$ is finite and $R / N(R)$ is a finite direct sum of absolutely algebraic fields of nonzero characteristic.

(3) $R=R_{1} \oplus \cdots \oplus R_{m} \oplus S$, where each $R_{i}$ is an absolutely algebraic field of nonzero characteristic and $S$ is a finite ring. 
Proof. (1) $\Rightarrow(2)$. Set $N=N(R)$ and choose $t \in \mathbb{Z}^{+}$such that $N^{t}=(0)$. To see that $N$ is finite, it suffices to see that $N^{i} / N^{i+1}$ is finite for $1 \leq i \leq t-1$; finiteness of $N^{i} / N^{i+1}$ follows because $N$ is a finitely generated $\pi[N]$-module, where $\pi$ is the prime subring of $R$, and $\pi[N] / N \simeq \pi /(\pi \cap N)$ is a finite ring. The ring $R / N$ is reduced, Noetherian and zero-dimensional, and hence is the direct sum of a finite family $\left\{F_{i}\right\}_{i=1}^{k}$ of fields; because $F_{i}$ is a residue field of $R / N$ for $1 \leq i \leq k$, it is absolutely algebraic of nonzero characteristic by Corollary 4.3.

(2) $\Rightarrow(3)$. If (2) holds, then $R$ has a composition series as an $R$-module, so $R$ is obviously Noetherian. Write $R$ as the direct sum of a finite family $\left\{\left(R_{i}, P_{i}\right)\right\}_{i=1}^{n}$ of primary rings. Then $N(R)=N\left(R_{1}\right) \oplus \cdots \oplus N\left(R_{n}\right)=P_{1} \oplus$ $\cdots \oplus P_{n}$. Since $R / N(R) \cong\left(R_{1} / P_{1}\right) \oplus \cdots \oplus\left(R_{n} / P_{n}\right)$, each $R_{i} / P_{i}$ is an absolutely algebraic field of nonzero characteristic. Since $N(R)$ is finite, each $P_{i}$ is finite, and to complete the proof, it suffices to show that $P_{i} \neq(0)$ implies that $R_{i} / P_{i}$ is finite, and hence $R_{i}$ is finite. Thus, let $k \geq 2$ be an integer such that $P_{i}^{k}=(0)$, but $P_{i}^{k-1} \neq(0)$. Choose a nonzero element $c \in P_{i}^{k-1}$. Then $R_{i} c$ is a onedimensional vector space over $R_{i} / P_{i}$. Since $R_{i} c \subseteq P_{i}, R_{i} c$ is finite, and hence $R_{i} / P_{i}$ is also finite.

(3) $\Rightarrow(1)$. If (3) is satisfied, then $S$ and each $R_{i}$ is hereditarily Noetherian, so Corollary 2.2 of $\left[\mathrm{GH}_{3}\right]$ implies that $R$ is hereditarily Noetherian.

Remark 4.11. Since, by Corollary 4.2, a hereditarily zero-dimensional ring is integral over its prime subring, conditions (1), (2), and (3) of Theorem 4.10 are also equivalent to each of the following.

(2) $\quad R=R_{1} \oplus \cdots \oplus R_{m} \oplus S$, where each $R_{i}$ is a field and $S$ is a finite ring.

(3)' The nilradical $N(R)$ of $R$ is finite and $\operatorname{Spec}(R)$ is finite.

If $R$ is a subring of a ring $S$, then $(R, S)$ is said to be a Noetherian pair if each subring of $S$ containing $R$ is Noetherian. In Theorem 4.12 we determine those zero-dimensional pairs that are Noetherian pairs.

Theorem 4.12. Let $(R, S)$ be a zero-dimensional pair. The following conditions are equivalent.

(1) $(R, S)$ is a Noetherian pair.

(2) $R$ is Noetherian and $S=S_{1} \oplus \cdots \oplus S_{n}$ is a finite direct sum of local Artinian rings such that each $S_{i}$ that is not a field is a finitely generated $R$-module.

(3) $R$ is Noetherian, $\operatorname{Spec}(S)$ is finite, and the nilradical $N$ of $S$ is a finitely generated $R$-module.

Proof. Corollary 4.2 implies that $S$ is integral over $R$. The equivalence of (1) and (2) follows from Proposition 1.2 and Theorem 1.6 of $\left[\mathrm{GH}_{3}\right]$. The equivalence of (1) and (3) follows from Theorem 1.5 of $\left[\mathrm{GH}_{3}\right]$, and the fact that if the nilradical $N$ of $S$ is a finitely generated ideal in $S$ and if $S / N$ is Noetherian, then $S$ is Noetherian.

\section{Directed unions of ARTINIAN SUbrings}

If $R$ is a commutative ring with prime subring $\pi$, then $R$ is the directed union of its family of subrings that are finitely generated over $\pi$. Each of these subrings is Noetherian, so $R$ is a directed union of Noetherian subrings. If 
$R$ is hereditarily zero-dimensional, it follows that $R$ is a directed union of Artinian subrings. We present in Propositions 5.1 and 5.2 examples of rings that are the directed union of Artinian subrings, but are not hereditarily zerodimensional. We then consider the condition on a ring $R$ that it is expressible as a directed union of Artinian subrings; in $\S 6$ we narrow our focus to the case where $R$ is a direct product of zero-dimensional rings. Initially we note that if $R$ is the directed union of a family $\left\{R_{\alpha}\right\}$ of subrings, each of dimension at most $n$, then $\operatorname{dim} R \leq n$; this follows since any chain $P_{0}<P_{1}<\cdots<P_{k}$ of prime ideals of $R$ contracts to a chain of distinct primes on some $R_{\alpha}$. Thus, a ring that is a directed union of Artinian subrings is zero-dimensional, and hereditarily zero-dimensional rings satisfy the given condition. We emphasize that in considering $R$ as the directed union of a family $\left\{R_{\alpha}\right\}$ of subrings in this section, we assume that each $R_{\alpha}$ contains the identity element of $R$.

Proposition 5.1. Let $F$ be a field, let $R=F^{\omega_{0}}$ be the countable direct product of $F$ with itself, and let $S$ be the subring of $R$ consisting of all sequences $\left\{a_{i}\right\}_{0}^{\infty}$ that are eventually constant. Then $S$ is a (von Neumann) regular ring that is the union of an ascending sequence of Artinian regular subrings containing $\pi$, the prime subring of $S$. The ring $S$ is hereditarily zero-dimensional if and only if $F$ is an absolutely algebraic field of characteristic $p \neq 0$.

Proof. For $j \geq 0$, let $S_{j}$ be the subring of $S$ consisting of all sequences $\left\{a_{i}\right\}_{0}^{\infty} \in S$ such that $a_{j}=a_{j+1}=\cdots$. Each $S_{j}$ contains $\pi$ (in fact, $S_{0}$ is the diagonal imbedding of $F$ in $R$ ), and to within isomorphism, $S_{j}=F^{j+1}$, an Artinian regular ring. Moreover, $S_{0} \subseteq S_{1} \subseteq \ldots, S=\bigcup_{j=0}^{\infty} S_{j}$, and $S$ is regular. According to Corollary 4.2, $S$ is hereditarily zero-dimensional if and only if char $S=\operatorname{char} F=p \neq 0$ and $S / \pi$ is integral. Clearly $S / \pi$ is integral if and only if each $S_{j} \simeq F^{j}$ is integral over $\pi \simeq G F(p)$ if and only if $F$ is algebraic over $G F(p)$-that is, if and only if $F$ is absolutely algebraic of characteristic $p \neq 0$.

Proposition 5.2. Let $F$ be a field, let $\alpha$ be an infinite cardinal, and let $R$ be the direct product of a copies of $F$ with itself. Let $S$ be the subring of $R$ consisting of all elements $\left\{a_{\beta}\right\}$ that have only finitely many distinct coordinates. Then $S$ is a regular ring expressible as the directed union of Artinian regular subrings, and $S$ is hereditarily zero-dimensional if and only if $F$ is an absolutely algebraic field of nonzero characteristic.

Proof. Let $A$ be a set of cardinality $\alpha$. We consider $R$ as the set of functions $f: A \rightarrow F$ under pointwise addition and multiplication and $S$ as $\{f \in R: f(A)$ is finite $\}$. Let $\mathscr{P}: A=A_{1} \cup \cdots \cup A_{t}$ be any finite partition of the set $A$. Then $S_{\mathscr{D}}=\left\{f \in R: f\right.$ is constant on each $\left.A_{i}\right\}$ is a subring of $S$ containing $\pi$, and $S_{\mathscr{P}} \simeq F^{s}$, where $s$ is the number of indices $i$ such that $A_{i}$ is nonempty. The family $\left\{S_{\mathscr{D}}\right\}$ is directed, for if $\mathscr{P}=\left\{A_{i}\right\}_{i=1}^{t}$ and $\mathscr{Q}=\left\{B_{i}\right\}_{i=1}^{v}$ are finite partitions of $A$, then $\mathscr{P} \cap \mathscr{Q}=\left\{A_{i} \cap B_{j}: 1 \leq i \leq t, 1 \leq j \leq v\right\}$ is a finite partition of $A$ and $S_{\mathscr{P}} \cup S_{\mathscr{Q}} \subseteq S_{\mathscr{P} \cap \mathscr{Q}}$. If $f \in S$, we can define a finite partition $\mathscr{P}$ on $A$ by considering the equivalence classes of the equivalence relation $\sim$ defined by $a \sim b$ if $f(a)=f(b)$. Clearly $f \in S_{\mathscr{P}}$, so $S$ is the directed union of the family of all such subrings $S_{\mathscr{P}}$. Each $S_{\mathscr{P}}$ is Artinian and regular, and hence $S$ is regular. According to Corollary 4.2, $S$ is hereditarily zero-dimensional if and only if $\operatorname{char} S=\operatorname{char} F=p \neq 0$ and $S / \pi$ is integral. Clearly $S / \pi$ is 
integral if and only if each $S_{\mathscr{P}} \simeq F^{j}$ is integral over $\pi \simeq G F(p)$ if and only if $F$ is algebraic over $G F(p)$-that is, if and only if $F$ is absolutely algebraic of characteristic $p \neq 0$.

We omit the straightforward proof of the following basic result.

Proposition 5.3. Suppose $R$ is a ring.

(1) If $R$ is a directed union of Artinian subrings, then each homomorphic image of $R$ has the same property.

(2) If $R=R_{1} \oplus \cdots \oplus R_{n}$, then $R$ is a directed union of Artinian subrings if and only if each $R_{i}$ has this property.

Theorem 5.4. Let $(S, M)$ be a zero-dimensional quasilocal ring, and let $A$ be an Artinian subring of $S$. Then there exists an Artinian subring $R$ of $S$ such that $R$ contains $A$, and $R \subseteq S$ is a zero-dimensional pair.

Proof. Let $P=M \cap A$. Since $A$ is a subring of $S$, and since the only idempotent elements in $S$ are 0 and $1, A$ is local with maximal ideal $P$. Let $\left\{x_{\lambda}\right\}$ be a subset of $S$ such that $\left\{x_{\lambda}+M\right\}$ is a transcendence basis for $S / M$ over $A / P$. Let $\left\{Y_{\lambda}\right\}$ be a set of indeterminates over $A$ and consider the natural map $\varphi: A\left[\left\{Y_{\lambda}\right\}\right] \rightarrow A\left[\left\{x_{\lambda}\right\}\right]$. If $f \in A\left[\left\{Y_{\lambda}\right\}\right]$ has unit content, then $\varphi(f) \notin M$, and hence $\varphi(f)$ is a unit of $S$. Let $U$ be the multiplicative system in $A\left[\left\{Y_{\lambda}\right\}\right]$ consisting of all polynomials of unit content. Then $\varphi(U)$ is a multiplicative system in $A\left[\left\{x_{\lambda}\right\}\right]$, and $\varphi$ extends to a surjection of $A\left[\left\{Y_{\lambda}\right\}\right]_{U}$ onto $R=A\left[\left\{x_{\lambda}\right\}\right]_{\varphi(U)} \subseteq S$. Since $A$ is Artinian, the ring $A\left[\left\{Y_{\lambda}\right\}\right]_{U}=A\left(\left\{Y_{\lambda}\right\}\right)$ is known to be zero-dimensional and Noetherian [N, p. 17, $\left.\mathrm{GH}_{1}\right]$, hence $\mathrm{Ar}-$ tinian, so $R$ is also Artinian. To see that $S$ is integral over $R$, we need only see that $S / M$ is integral over $R /(M \cap R)$; this follows since, by choice of $\left\{x_{\lambda}\right\}$, $S / M$ is integral over $A\left[\left\{x_{\lambda}\right\}\right] /\left(M \cap A\left[\left\{x_{\lambda}\right\}\right]\right)$ and $A\left[\left\{x_{\lambda}\right\}\right] \subseteq R$.

Corollary 5.5. If $S$ is a zero-dimensional ring with finite spectrum, then there exists an Artinian subring $R$ of $S$ such that $(R, S)$ is a zero-dimensional pair. In particular, $S$ is a directed union of Artinian subrings.

Proof. $S$ is the direct sum of a finite family $\left\{\left(S_{i}, M_{i}\right)\right\}_{i=1}^{t}$ of zero-dimensional quasilocal rings. If $A_{i}$ is an Artinian subring of $S_{i}$ such that $\left(A_{i}, S_{i}\right)$ is a zerodimensional pair, then $A=A_{1} \oplus \cdots \oplus A_{t}$ is Artinian, and since $S$ is integral over $A,(A, S)$ is a zero-dimensional pair. Thus, it suffices to prove Corollary 5.5 in the case where $(S, M)$ is quasilocal, and in light of Theorem 5.4, this case follows if we show that $S$ admits an Artinian subring. If $\operatorname{char}(S / M)=0$, then $S$ contains $\mathbb{Z}$, and since $\mathbb{Z} \cap M=(0), \mathbb{Q}$ is an Artinian subring of $S$. If $\operatorname{char}(S / M)=p \neq 0$, then $p$ is nilpotent so the prime subring of $S$ is a finite, hence Artinian, subring of $S$. In any event, $S$ contains an Artinian subring, and this completes the proof of Corollary 5.5.

The proof of Corollary 5.5 uses the fact that if a zero-dimensional ring $S$ is integral over a Noetherian subring, then $S$ is the directed union of a family of Artinian subrings. The last result of this section, Example 5.6, shows that the converse of the preceding statement fails. The construction used in Example 5.6 is a modification of that of Proposition 5.1.

Example 5.6. Let $F$ be a field that has finite transcendence degree over its prime subfield $\pi$, let $\left\{X_{i}\right\}_{i=1}^{\infty}$ be a set of indeterminates over $F$, and for each 
positive integer $n$, let $F_{n}=F\left(X_{1}, X_{2}, \ldots, X_{n}\right)$. Let $R$ be the subring of $\prod_{i=1}^{\infty} F_{i}$ consisting of all sequences $\left\{a_{i}\right\}_{i=1}^{\infty}$ that are eventually constant. We show that $R$ is a von Neumann regular ring expressible as the union of an ascending sequence of Artinian rings, but that $R$ is not integral over any subring with Noetherian spectrum. The first assertions of the preceding sentence follow as in the proof of Proposition 5.1. To wit, if $R_{n}$ is the set of sequences $\left\{a_{i}\right\}_{i=1}^{\infty}$ in $R$ such that $a_{n}=a_{n+1}=\ldots$, then $R_{n} \simeq F_{1} \oplus F_{2} \oplus \cdots \oplus F_{n}, R_{1} \subseteq R_{2} \subseteq \ldots$, and $R=\bigcup_{i=1}^{\infty} R_{i}$. Suppose $A$ is a subring of $R$ such that $R / A$ is integral. To show that $A$ does not have Noetherian spectrum, we analyze $\operatorname{Spec}(R)$. Thus, for $i \in \mathbb{Z}^{+}$, we let $e_{i}=\left\{\delta_{i j}\right\}_{j=1}^{\infty} \in R$, where $\delta_{i j}$ is the Kronecker delta. If $P \in \operatorname{Spec}(R)$, then either (1) $e_{i} \notin P$ for some $i$, or (2) $e_{i} \in P$ for all $i$.

If (1) holds, then $1-e_{i} \in P$, and since $1-e_{i}$ generates a maximal ideal of $R$ with $R /\left(1-e_{i}\right) \simeq F_{i}$, it follows that $P=\left(1-e_{i}\right)$ in this case.

If (2) holds, then $P \supseteq P_{\infty}$, the ideal of $R$ consisting of all sequences that are eventually 0 . $P_{\infty}$ is the kernel of the surjective homomorphism $\varphi: R \rightarrow \bigcup_{i=1}^{\infty} F_{i}$ defined by taking $\varphi\left(\left\{a_{i}\right\}\right)$ to be the eventual value of the sequence $\left\{a_{i}\right\}$. Hence $P_{\infty}$ is a maximal ideal of $R, P=P_{\infty}$, and $R / P_{\infty} \simeq F\left(\left\{X_{i}\right\}_{1}^{\infty}\right)$. Clearly $P_{\infty}$ is not finitely generated. We observe that $P_{\infty}$ is the only prime ideal of $R$ whose associated residue field has infinite transcendence degree over its prime subfield $\pi$.

Now $R / P_{\infty}$ is integral over $A /\left(P_{\infty} \cap A\right)$, so $A /\left(P_{\infty} \cap A\right)$ has infinite transcendence degree over $\pi$. Consequently, $P_{\infty}$ is the only prime of $R$ lying over $P_{\infty} \cap A$ in $A$. Therefore $P_{\infty}=\sqrt{\left(P_{\infty} \cap A\right) R}$, and because $R$ is von Neumann regular, $\sqrt{\left(P_{\infty} \cap A\right) R}=\left(P_{\infty} \cap A\right) R$. Thus $P_{\infty}=\left(P_{\infty} \cap A\right) R$, so $P_{\infty} \cap A$ is not finitely generated since $P_{\infty}$ is not finitely generated. Because $R / A$ is integral, the ring $A$ is zero-dimensional and reduced, hence von Neumann regular. Therefore $A$ does not have Noetherian spectrum since $A$ is not Noetherian. This completes the presentation of Example 5.6.

\section{6. $\prod R_{\alpha}$ AS A DIRECTED UNION OF ARTINIAN SUBRINGS}

In this section we consider the problem of determining conditions under which a direct product $\Pi R_{\alpha}$ is expressible as a directed union of Artinian subrings. Obvious necessary conditions on $\prod R_{\alpha}$ are that it should be zerodimensional and that each $R_{\alpha}$ is so expressible. Hence each $R_{\alpha}$ is zerodimensional and Theorem 3.4 shows that the set $\left\{\alpha \in A: \eta\left(R_{\alpha}\right)>k\right\}$ is finite for some $k \in \mathbb{Z}^{+}$. To obtain a third necessary condition on $\prod R_{\alpha}$, we observe that if $S$ is any ring expressible as a union of subrings with finite spectra (hence if $S$ is expressible as the union of Artinian subrings), then the set $\mathscr{C}(S)=\{\operatorname{char}(S / P): P \in \operatorname{Spec}(S)\}$ is finite. To verify this assertion, we note that if $\operatorname{char}(S / P) \neq 0$, then $\operatorname{char}(S / P)=\operatorname{char}(R /(R \cap P))$ for each subring $R$ of $S$ containing the prime subring of $S$; hence $\mathscr{C}(S)$ is finite because there exists such an $R$ that has finite spectrum. In particular, if $\prod R_{\alpha}$ is a union of subrings with finite spectrum, then the set $\bigcup_{\alpha \in A} \mathscr{C}\left(R_{\alpha}\right)$ is finite.

Theorem 6.7 provides a definitive answer to the main question addressed in this section. Theorem 6.7 is preceded by three special cases thereof. These special cases are covered in Theorems 6.3-6.5; these three theorems treat a direct product $\prod D_{\alpha}$ of integral domains: they state that, under appropriate hypotheses, $\prod D_{\alpha}$ is not a directed union of Artinian subrings. In fact, the 
conclusions of Theorems 6.3-6.5 are the stronger statement that $\prod D_{\alpha}$ is not a directed union of subrings with finite spectra. Lemma 6.1 is a key result in the proofs of Theorems 6.3-6.5.

Lemma 6.1. Assume that $R$ is a subring of the ring $T$ and that $\left\{N_{\lambda}\right\}$ is a family of prime ideals of $T$, each of which lies over the prime ideal $P$ of $R$, where $R / P$ is a normal domain. For $b \in T$, let $b_{\lambda}$ denote the image of $b$ in $T / N_{\lambda}$, and if $b_{\lambda}$ is integral over $R / P$, denote by $f_{\lambda}(X)$ the minimal polynomial for $b_{\lambda}$ over the quotient field of $R / P$. Since $R / P$ is normal, $f_{\lambda}(X) \in(R / P)[X]$. If there exists $b \in T$ such that the corresponding set $\left\{f_{\lambda}(X)\right\} \subseteq(R / P)[X]$ is infinite, then for any subring $S$ of $T$ containing $R[b]$, the set $\left\{N_{\lambda} \cap S\right\}$ is infinite. In particular, $T$ is not the union of a family $\left\{S_{\alpha}\right\}$ of subrings, where each $S_{\alpha}$ contains $R$ and has finite spectrum.

Proof. Choose a sequence $\left\{f_{i}(X)\right\}_{i=1}^{\infty}$ of distinct elements of $\left\{f_{\lambda}(X)\right\}$ and assume that $N_{i} \in\left\{N_{\lambda}\right\}$ is such that the image $b_{i}$ of $b$ in $T / N_{i}$ has minimal polynomial $f_{i}(X) \in(R / P)[X]$. Suppose $S$ is a subring of $T$ containing $R[b]$ and let $Q_{i}=N_{i} \cap S$. Then $R / P \subseteq S / Q_{i} \subseteq T / N_{i}$ and $b_{i} \in S / Q_{i}$. If $Q_{i}$ and $Q_{j}$ were equal for some $i \neq j$, it would follow that $f_{i}(X)=f_{j}(X)$ in $(R / P)[X]$, contrary to the choice of the sequence $\left\{f_{i}\right\}$. We conclude that $Q_{i} \neq Q_{j}$ for $i \neq j$, and this establishes the desired assertion.

We note that the proof of Lemma 6.1 shows that, in fact, $\left|\left\{N_{\lambda} \cap S\right\}\right| \geq$ $\left|\left\{f_{\lambda}(X)\right\}\right|$. The next result is an analogue of Proposition 5.2; again we omit the straightforward proof.

Proposition 6.2. Suppose $R$ is a ring.

(1) If $R$ is a (directed) union of (zero-dimensional) subrings with finite spectra, then each homomorphic image of $R$ has the same property.

(2) If $R=R_{1} \oplus \cdots \oplus R_{n}$, then $R$ is a (directed) union of (zero-dimensional) subrings with finite spectra if and only if each $R_{i}$ is a (directed) union of (zerodimensional) subrings with finite spectra.

Theorem 6.3. Suppose $D_{\alpha}$ is an integral domain of characteristic zero for each $\alpha \in A$, an infinite set. Then $T=\prod_{\alpha \in A} D_{\alpha}$ is not the union of a family of subrings, each with finite spectrum.

Proof. Since the set $A$ is infinite, and since it suffices, by (6.2), to show that a direct summand of $T$ is not such a union, we may assume that $A=\mathbb{Z}^{+}$ and $T=\prod_{i=1}^{\infty} D_{i}$. Denote by $R \simeq \mathbb{Z}$ the prime subring of $T$. We apply Lemma 6.1 to the element $b=\{n\}_{n=1}^{\infty} \in T$ and to the prime ideals $P_{n}$ of $T$, where $P_{n}$ consists of those elements of $T=\prod_{i=1}^{\infty} D_{i}$ with $n$th coordinate 0 . The image of $b$ in $T / P_{n}$ is $n$, and the minimal polynomial for $n$ over $R /\left(P_{n} \cap R\right)=R /(0) \simeq \mathbb{Z}$ is $X-n$. We conclude from (6.1) that $T$ is not the union of any family of subrings, each with finite spectrum.

Theorem 6.4. Let $\left\{D_{\alpha}\right\}_{\alpha \in A}$ be a family of integral domains, all of the same characteristic $p>0$. If $D_{\alpha}$ is not algebraic over its prime subfield $\mathbb{Z}_{p}$ for infinitely many elements $\alpha \in A$, then $\prod D_{\alpha}$ is not the directed union of a family of subrings, each with finite spectrum.

Proof. As in the proof of Theorem 6.3, it suffices to consider the case where $A=\mathbb{Z}^{+}$and $D_{i}$ contains an element $b_{i}$ transcendental over $\mathbb{Z}_{p}$ for each $i \in A$. Consider the elements $b=\left\{b_{i}\right\}_{i=1}^{\infty}$ and $c=\left\{b_{i}^{i}\right\}_{i=1}^{\infty}$ in $T=\prod_{1}^{\infty} D_{i}$. 
Let $R=\pi[c]$, where $\pi \simeq \mathbb{Z}_{p}$ is the prime subring of $T$. Assume that $T$ is the directed union of a family $\left\{R_{\beta}\right\}$ of subrings of $T$. Then there exists $S \in\left\{R_{\beta}\right\}$ such that both $b$ and $c$ belong to $S$. Moreover, $R=\pi[c] \subseteq S$ since $\pi \subseteq S$. Let $N_{i}$ be the prime ideal of $T$ consisting of those sequences with $i$ th coordinate 0 . Then $N_{i} \cap R=(0)$ and the image of $R$ in $T / N_{i} \simeq D_{i}$ is $\mathbb{Z}_{p}\left[b_{i}^{i}\right]$, a simple transcendental extension of the field $\mathbb{Z}_{p}$, and hence a normal domain. Let $N_{i} \cap S=Q_{i}$. The image of $b \in S$ in $S / Q_{i} \subseteq T / N_{i}=D_{i}$ is $b_{i}$. The minimal polynomial for $b_{i}$ over $\mathbb{Z}_{p}\left[b_{i}^{i}\right]$ is $X^{i}-b_{i}^{i} \in \pi\left[b_{i}^{i}\right][X]$. Lemma 6.1 implies that there are infinitely many distinct $Q_{i}$ (in fact, the ideals $Q_{i}$ are distinct), and hence that $\operatorname{Spec} S$ is infinite. We conclude that $T$ is not the directed union of subrings $R_{\alpha}$, where $\operatorname{Spec} R_{\alpha}$ is finite.

Theorem 6.5. Let $\left\{D_{\alpha}\right\}_{\alpha \in A}$ be a family of integral domains, all of the same characteristic $p>0$, and let $F_{\alpha}$ denote the algebraic closure of $\mathbb{Z}_{p}$ in $D_{\alpha}$. If for each $n \in \mathbb{Z}^{+}$the set $\left\{\alpha \in A:\left|F_{\alpha}\right| \geq p^{n}\right\}$ is infinite, then $T=\prod D_{\alpha}$ is not a union of subrings with finite spectra.

Proof. Again we assume without loss of generality that $A=\mathbb{Z}^{+}$and that $\left|F_{n}\right| \geq$ $p^{n}$ for each $n$. Choose $b_{n} \in F_{n} \subseteq D_{n}$ of degree at least $n$ over $\mathbb{Z}_{p}$ for each $n \in \mathbb{Z}^{+}$, let $b=\left\{b_{i}\right\}_{i=1}^{\infty}$, and let $R \simeq \mathbb{Z}_{p}$ be the prime subring of $T$. Applying Lemma 6.1 to $R, T, b$, and to the family $\left\{N_{i}\right\}_{i=1}^{\infty}$ of ideals of $T$, with $N_{i}$ as defined in the proof of (6.4), we obtain the desired conclusion, as in the proof of Theorems 6.3 and 6.4 .

We now direct our attention to the general direct product $\prod R_{\alpha}$; Theorem 6.6 considers the case where each $R_{\alpha}$ is quasilocal.

Theorem 6.6. Let $\left\{\left(R_{\alpha}, M_{\alpha}\right\}_{\alpha \in A}\right.$ be a family of zero-dimensional quasilocal rings, let $K_{\alpha}=R_{\alpha} / M_{\alpha}$, and let $T=\prod R_{\alpha}$. The following conditions are equivalent.

(1) $T$ is a directed union of Artinian subrings.

(2) $T$ is a directed union of zero-dimensional subrings with finite spectra.

(3) $T$ is a directed union of subrings with finite spectra.

(4) There exists $n \in \mathbb{Z}^{+}$such that the set $\left\{\alpha \in A: \eta\left(R_{\alpha}\right)>n\right.$ or $\left.\left|K_{\alpha}\right|>n\right\}$ is a finite set.

Proof. The implications $(1) \Rightarrow(2)$ and $(2) \Rightarrow(3)$ are clear.

(3) $\Rightarrow(4)$. If (3) holds, then the introductory paragraph of this section shows that the set $H=\left\{\operatorname{char} K_{\alpha}\right\}_{\alpha \in A}$ is finite. If $0 \in H$, then Theorem 6.3 implies that $\left\{\alpha \in A\right.$ : char $\left.K_{\alpha}=0\right\}$ is finite, and if $p \in H, p>0$, Theorems 6.4 and 6.5 imply that for some $n \in \mathbb{Z}^{+}$the set $\left\{\alpha \in A: \operatorname{char} K_{\alpha}=p\right.$ and $\left.\left|K_{\alpha}\right|>p^{n}\right\}$ is also finite. Consequently, if (4) is not satisfied, then there exists a sequence $\left\{R_{i}\right\}_{i=1}^{\infty} \subseteq\left\{R_{\alpha}\right\}$ such that $\eta\left(R_{i}\right)>i$ for each $i$. But then Theorem 3.4 and Corollary 3.6 show that $T$ has a homomorphic image that is an infinite product $\prod_{i=1}^{\infty} D_{i}$ of integral domains $D_{i}$ of positive dimension. Since $\prod_{i=1}^{\infty} D_{i}$ is a union of subrings with finite spectra, then as we observed at the beginning of this section, the set $\bigcup_{i=1}^{\infty} \mathscr{C}\left(D_{i}\right)$ is a finite set. Hence infinitely many of the domains $D_{i}$ have the same characteristic. But then Theorems (6.3)-(6.5) yield the contradiction that $\prod_{i=1}^{\infty} D_{i}$, and hence $T$, is not the directed union of subrings with finite spectra.

(4) $\Rightarrow$ (1). Let $L=\left\{\alpha \in A: \eta\left(R_{\alpha}\right)>n\right.$ or $\left.\left|K_{\alpha}\right|>n\right\}=\left\{\alpha_{1}, \ldots, \alpha_{t}\right\}$ and let $B=A \backslash L$. Then $T=\left(\prod_{\alpha \in B} R_{\alpha}\right) \oplus R_{\alpha_{1}} \oplus \cdots \oplus R_{\alpha_{t}}$. Each $R_{\alpha}$ is hereditarily 
zero-dimensional by Corollary 4.3 , so Theorem 4.9 implies that $\prod_{\alpha \in B} R_{\alpha}$ is hereditarily zero-dimensional. Thus $\prod_{\alpha \in B} R_{\alpha}$ and, by Theorem 5.4, each $R_{\alpha_{i}}$ is a directed union of Artinian subrings; by Proposition 5.3, $T$ shares the same property, and this completes the proof of Theorem 6.6.

Theorem 6.7. Let $\left\{R_{\alpha}\right\}_{\alpha \in A}$ be a family of rings and let $T=\prod R_{\alpha}$. Then $T$ is a directed union of Artinian subrings (respectively, of zero-dimensional subrings with finite spectra) if and only if each $R_{\alpha}$ is so expressible and there exists $n \in \mathbb{Z}^{+}$such that the set $\left\{\alpha \in A: \eta\left(R_{\alpha}\right)>n\right.$ or $R_{\alpha}$ has a residue field $K_{\alpha}$ with $\left.\left|K_{\alpha}\right|>n\right\}$ is finite.

Proof. We give the proof in the case of directed unions of Artinian subrings; the proof in the case of zero-dimensional subrings with finite spectra is quite similar.

$(\Rightarrow)$. The assertion concerning $R_{\alpha}$ follows from (5.3). Suppose the condition concerning existence of $n \in \mathbb{Z}^{+}$fails. Then either

(i) there exists a sequence $\left\{R_{i}\right\}_{i=1}^{\infty} \subseteq\left\{R_{\alpha}\right\}$ such that $R_{i}$ has a residue field $K_{i}$ with $\left|K_{i}\right|>i$, or else

(ii) there exists a sequence $\left\{R_{i}\right\}_{i=1}^{\infty} \subseteq\left\{R_{\alpha}\right\}$ such that $\eta\left(R_{i}\right)>i$ for each $i$.

If (i) holds, then Theorem 6.6 shows that the homomorphic image $\prod_{i=1}^{\infty} K_{i}$ of $T=\prod R_{\alpha}$ is not expressible as a directed union of Artinian subrings, and hence neither is $T$. If (ii) holds, then as in the proof of (6.6), $T$ is infinitedimensional, and hence not a directed union of Artinian subrings.

$(\Leftarrow)$. Let $B=\left\{\alpha \in A: \eta\left(R_{\alpha}\right) \leq n\right.$ and each residue field of $R_{\alpha}$ has cardinality $\leq n\}$. By hypothesis, $A \backslash B$ is a finite set. Since each $R_{\alpha}$ is a directed union of Artinian subrings and since $T$ is the direct product of $\prod_{\alpha \in B} R_{\alpha}$ and the finite family $\left\{R_{\alpha}\right\}_{\alpha \in A \backslash B}$, it suffices to show that $\prod_{\alpha \in B} R_{\alpha}$ is a directed union of Artinian subrings. For each $R \in\left\{R_{\alpha}\right\}_{\alpha \in B}, R$ is imbedded in $\prod\left\{R_{M}: M\right.$ is a maximal ideal of $\left.R\right\}$, and hence $\prod_{\alpha \in B} R_{\alpha}$ is imbedded in $\prod_{\alpha \in B}\left(\prod_{M \in M \operatorname{Spec}\left(R_{\alpha}\right)}\left(R_{\alpha}\right)_{M}\right)$. Corollary 4.3 and Theorem 4.9 show that the latter of these two rings is hereditarily zero-dimensional, and hence $\prod_{\alpha \in B} R_{\alpha}$ is also hereditarily zero-dimensional. Thus $\prod_{\kappa \in B} R_{\alpha}$ is a directed union of Artinian subrings, as we wished to show.

Theorem 6.7 admits a parallel statement for rings expressible as a directed union of subrings with finite spectra. We omit the proof of this result because of its similarity to the proof of Theorem 6.7.

Theorem 6.8. Let $\left\{R_{\alpha}\right\}_{\alpha \in A}$ be a family of rings and let $T=\prod_{\alpha \in A} R_{\alpha}$. Then $T$ is a directed union of subrings with finite spectra if and only if each $R_{\alpha}$ is so expressible and there exists $n \in \mathbb{Z}^{+}$such that the set $\left\{\alpha \in A: \operatorname{dim}\left(R_{\alpha}\right)>0\right.$ or $\eta\left(R_{\alpha}\right)>n$ or $R_{\alpha}$ has a residue field $K_{\alpha}$ with $\left.\left|K_{\alpha}\right|>n\right\}$ is finite.

Remark 6.9. If a ring $R$ is expressible as the directed union of a family $\left\{R_{\alpha}\right\}_{\alpha \in A}$ of zero-dimensional subrings with finite spectra, it would be interesting to know whether $R$ is then necessarily the directed union of a family of Artinian subrings. In this connection we note that Corollary 5.5 shows that each $R_{\alpha}$ is a directed union of Artinian subrings, so $R$ is at least a set-theoretic union of Artinian subrings. A factor that comes into play here is that although $R_{\alpha}$ is 
known by Corollary 5.5 to be a directed union of Artinian subrings, it is unclear as to whether the family of all Artinian subrings of $R_{\alpha}$ is directed. It would be interesting to know necessary and sufficient conditions on a zero-dimensional quasilocal ring $S$ in order that the family of all Artinian subrings of $S$ forms a directed family.

Remark 6.10. In relation to directed unions, a question we have considered but not resolved is the following. If $T$ is a zero-dimensional ring with nilradical $N$ and if $T / N$ is a directed union of Artinian subrings, does $T$ have the same property? Corollary 5.5 yields an affirmative answer in the case where $T$ has finite spectrum.

Added in Proof. In connection with (6.10) we can show that $T$ is a set-theoretic union of a family $\left\{S_{i}\right\}$ of Artinian subrings. We can obtain such a family $\left\{S_{i}\right\}$ with the property that each finite subset of $T$ is contained in some $S_{i}$, but do not know whether there exists a directed family.

\section{REFERENCES}

[AG] J. T. Arnold and R. Gilmer, Dimension theory of commutative rings without identity, J. Pure Appl. Algebra 5 (1974), 209-231.

[BW] H. S. Butts and L. I. Wade, Two criteria for Dedekind domains, Amer. Math. Monthly 73 (1966), 14-21.

[CC] P.-J. Cahen and J.-L. Chabert, Coefficients et valeurs d'un polynome, Bull. Sci. Math. 95 (1971), 295-304.

[C] J.-L. Chabert, Un anneau de Prüfer, J. Algebra 107 (1987), 1-16.

[CL] K. L. Chew and S. Lawn, Residually finite rings, Canad. J. Math. 22 (1970), 91-101.

$\left[\mathrm{G}_{1}\right] \quad$ R. Gilmer, A note on two criteria for Dedekind domains, Enseign. Math. 13 (1967), 253-256.

$\left[\mathrm{G}_{2}\right] \quad$, Integral domains with Noetherian subrings, Comment. Math. Helv. 45 (1970), 129134.

$\left[\mathrm{G}_{3}\right] \ldots$ On polynomial rings over a Hilbert ring, Michigan Math. J. 18 (1971), 205-212.

$\left[\mathrm{G}_{4}\right] \_$, Multiplicative ideal theory, Marcel Dekker, New York, 1972.

$\left[\mathrm{GH}_{1}\right]$ R. Gilmer and W. Heinzer, The Noetherian property for quotient rings of infinite polynomial rings, Proc. Amer. Math. Soc. 76 (1979), 1-7.

$\left[\mathrm{GH}_{2}\right]+$ Some countability conditions on commutative ring extensions, Trans. Amer. Math. Soc. 264 (1981), 217-234.

$\left[\mathrm{GH}_{3}\right] \ldots$ Noetherian pairs and hereditarily Noetherian rings, Arch. Math. 41 (1983), 131-138.

$\left[\mathrm{GH}_{4}\right] \ldots$, On the imbedding of a direct product into a zero-dimensional commutative ring, Proc. Amer. Math. Soc. 106 (1989), 631-637.

[H] J. Huckaba, Commutative rings with zero divisors, Dekker, 1988.

[HL] P. Huet and D. Lazard, Dominions des anneaux commutatifs, Bull. Sci. Math. 94 (1970), 193-199.

[K] E. Kunz, Introduction to commutative algebra and algebraic geometry, Birkhäuser, 1985.

[LM] K. Levitz and J. L. Mott, Rings with finite norm property, Canad. J. Math. 24 (1972), 557-565.

[M] P. Maroscia, Sur les anneaux de dimension zero, Atti Accad. Naz. Lincei Rend Cl. Sci. Fis. Mat. Natur. 56 (1974), 451-459.

[Mc] D. L. McQuillan, On Prüfer domains of polynomials, J. Reine Angew. Math. 358 (1985), 162-178.

[N] M. Nagata, Local rings, Interscience, 1962. 
[SSY] F. Shibata, T. Sugatani, and K. Yoshida, Note on rings of integer-valued polynomials, C. R. Math. Rep. Acad. Sci. Canada 8 (1986), 297-301.

[ZS] O. Zariski and P. Samuel, Commutative algebra, vol. I, Springer-Verlag, 1975.

Department of Mathematics, Florida State University, Tallassee, Florida 32306 3027

E-mail address: glimer@gauss.math.fsu.edu

Department of Mathematics, Purdue University, West Lafayette, Indiana 47907

E-mail address: heinzer@math.purdue.edu 